PDES, SUBMANIFOLDS AND

AFFINE DIFFERENTIAL GEOMETRY

BANACH CENTER PUBLICATIONS, VOLUME 69

INSTITUTE OF MATHEMATICS

POLISH ACADEMY OF SCIENCES

WARSZAWA 2005

\title{
AFFINE MAXIMAL HYPERSURFACES
}

\author{
AN-MIN LI and FANG JIA \\ Department of Mathematics, Sichuan University \\ Chengdu, Sichuan, P.R. China \\ E-mail: math-li@yahoo.com.cn, galaxyly2000@yahoo.com.cn
}

\begin{abstract}
This paper is part of the autumn school on "Variational problems and higher order PDEs for affine hypersurfaces". We discuss affine Bernstein problems and complete constant mean curvature surfaces in equiaffine differential geometry.
\end{abstract}

\section{Contents}

1. Affine Weierstrass representation

2. Proof of Calabi's Affine Bernstein Conjecture

3. Euclidean complete affine surfaces with constant affine mean curvature

1. Affine Weierstrass representation. We use the notation for equiaffine hypersurfaces in real affine space $A^{n+1}$ from [L-S-Z]. Let $M$ be a connected, oriented differentiable manifold, and let $x: M \rightarrow A^{n+1}$ be a locally strongly convex hypersurface. If the unimodular-affine mean curvature $L_{1}$ satisfies $L_{1}=0$ on $M$ then $x(M)$ is called an affine maximal hypersurface.

The conormal field $U$ satisfies the PDE $\Delta U+n L_{1} U=0$; this implies the following

THEOREM 1.1. Let $x: M \rightarrow A^{n+1}$ be a locally strongly convex hypersurface, given as the graph of a function $f ; x$ is an affine maximal hypersurface (which means $L_{1} \equiv 0$ on $M$ ) if and only if $f$ satisfies the PDE

$$
\Delta\left\{\left[\operatorname{det}\left(\frac{\partial^{2} f}{\partial x^{j} \partial x^{i}}\right)\right]^{-1 /(n+2)}\right\}=0,
$$

2000 Mathematics Subject Classification: 53A15, 49Q05, 53C42.

Key words and phrases: equiaffine maximal hypersurfaces, affine Bernstein problems.

Both authors are partially supported by 973 project, NSFC 10271083 grant and a ChineseGerman exchange project of NSFC and DFG; conference support by VolkswagenStiftung.

The paper is in final form and no version of it will be published elsewhere. 
where the Laplacian, in local coordinates, satisfies:

$$
\Delta=\frac{1}{\sqrt{\operatorname{det}\left(G_{k l}\right)}} \sum_{i, j=1}^{n} \frac{\partial}{\partial x^{i}}\left(G^{i j} \sqrt{\operatorname{det}\left(G_{k l}\right)} \frac{\partial}{\partial x^{j}}\right) .
$$

ExAmple 1.2. Any parabolic affine hypersphere is an affine maximal hypersurface. In particular, the elliptic paraboloid

$$
x^{n+1}=\frac{1}{2}\left[\left(x^{1}\right)^{2}+\cdots+\left(x^{n}\right)^{2}\right], \quad\left(x^{1}, \ldots, x^{n}\right) \in A^{n}
$$

is an affine-complete affine maximal hypersurface.

An affine analogue of the Weierstrass representation. Let $V$ be the $\mathbb{R}$-vector space associated to $A^{n+1}$. We consider a Euclidean inner product $(\cdot, \cdot): V \times V \rightarrow \mathbb{R}$ with associated normed determinant forms on $V$ and its dual space $V^{*}$; we use the same notation Det. For simplicity, we study maximal surfaces in $\mathbb{R}^{3}$. Let $x: M \rightarrow \mathbb{R}^{3}$ be a locally strongly convex surface. Choose isothermal parameters $u, v$ on $M$ with respect to the Blaschke metric $G$, and let $e_{1}=\partial_{u} x=x_{u}, e_{2}=\partial_{v} x=x_{v}$, and denote $\partial U / \partial u=: U_{u}, \partial U / \partial v=: U_{v}$. Then $G_{11}=G_{22}=: F>0, G_{12}=G_{21}=0$. We have

$$
\begin{gathered}
\left(U, x_{u}\right)=0, \quad\left(U_{u}, x_{u}\right)=-F, \quad\left(U_{v}, x_{u}\right)=0, \\
\left(U, x_{v}\right)=0, \quad\left(U_{u}, x_{v}\right)=0, \quad\left(U_{v}, x_{v}\right)=-F \\
(U, Y)=1, \quad\left(U_{u}, Y\right)=0, \quad\left(U_{v}, Y\right)=0
\end{gathered}
$$

where $Y$ denotes the affine normal.

We use the cross product construction; then (see [L-S-Z], p. 3)

$$
\begin{gathered}
x_{u}=\lambda\left[U, U_{v}\right], \quad x_{v}=\mu\left[U, U_{u}\right], \\
\operatorname{Det}\left(x_{u}, x_{v}, Y\right) \operatorname{Det}\left(U_{u}, U_{v}, U\right)=F^{2},
\end{gathered}
$$

where $\lambda, \mu$ are differentiable functions. Since

$$
\operatorname{Det}\left(x_{u}, x_{v}, Y\right)=\left|\operatorname{det}\left(h_{i j}\right)\right|^{1 / 4}=F,
$$

we have

$$
\operatorname{Det}\left(U_{u}, U_{v}, U\right)=F
$$

Then

$$
-F=\left(U_{u}, x_{u}\right)=-\lambda \operatorname{Det}\left(U_{u}, U_{v}, U\right)=-\lambda F .
$$

It follows that $\lambda=1$. Similarly, we have $\mu=-1$.

Thus we obtain the following affine analogue of the well known Weierstrass representation for Euclidean minimal surfaces:

$$
x=\int\left[U, U_{v}\right] d u-\left[U, U_{u}\right] d v .
$$

If $x(M)$ is an affine maximal surface then

$$
\Delta U=0,
$$

where, in the given coordinate system, the Laplacian reads:

$$
\Delta=\frac{1}{\operatorname{Det}\left(U_{u}, U_{v}, U\right)}\left(\frac{\partial^{2}}{\partial u^{2}}+\frac{\partial^{2}}{\partial v^{2}}\right) .
$$


It follows that the components $U^{1}(u, v), U^{2}(u, v)$ and $U^{3}(u, v)$ of $U$ are harmonic functions.

Conversely, given a triple of functions

$$
U=\left(U^{1}(u, v), U^{2}(u, v), U^{3}(u, v)\right)
$$

defined on $\Omega \subset \mathbb{R}^{2}$, satisfying the two conditions:

(i) $U^{1}, U^{2}, U^{3}$ are harmonic with respect to the canonical metric of $\mathbb{R}^{2}$;

(ii) $\operatorname{Det}\left(U_{u}, U_{v}, U\right)>0$ in $\Omega$, where $\Omega$ is a simply connected domain and $\left(u_{0}, v_{0}\right),(u, v) \in \Omega$.

Then we can construct an affine maximal surface $x: \Omega \rightarrow A^{3}$ as follows:

$$
x=\int_{\left(u_{0}, v_{0}\right)}^{(u, v)}\left[U, U_{v}\right] d u-\left[U, U_{u}\right] d v .
$$

The surface is well defined because the integrability conditions are satisfied:

$$
\left[U, U_{v}\right]_{v}+\left[U, U_{u}\right]_{u}=\left[U, U_{u u}+U_{v v}\right]=0 .
$$

From the point of view of local differential geometry the formula (1.2) admits a construction of any affine maximal surface.

In the following we give some further examples of affine maximal surfaces.

Example 1.3. Consider $\Omega:=\mathbb{R}^{2}$ and define $U: \mathbb{R}^{2} \rightarrow \mathbb{R}^{3}$ by $U:=(1, u, v)$; we get

$$
x=\left(\frac{1}{2}\left(u^{2}+v^{2}\right),-u,-v\right),
$$

which is an elliptic paraboloid.

Example 1.4. Consider $\Omega:=\left\{(u, v) \in \mathbb{R}^{2} \mid u>0\right\}$ and define $U: \Omega \rightarrow \mathbb{R}^{3}$ by $U:=$ $\left(1, u^{2}-v^{2}, v\right)$, then

$$
\operatorname{Det}\left(U_{u}, U_{v}, U\right)=2 u \text {. }
$$

The construction above gives

$$
x=\left(\frac{1}{3}\left(u^{3}+u v^{2}\right),-u,-2 u v\right), u>0 .
$$

EXAmple 1.5. Let $\Omega:=\left\{(u, v) \in \mathbb{R}^{2} \mid u>0, v<0\right\}$ and define $U:=(u, v, 2 u v)$, then

$$
\begin{gathered}
\operatorname{Det}\left(U_{u}, U_{v}, U\right)=-2 u v, \\
x=\left(-\frac{2}{3} v^{3},-\frac{2}{3} u^{3}, \frac{1}{2}\left(u^{2}+v^{2}\right)\right), \quad u>0, v<0 .
\end{gathered}
$$

Affine completeness and Euclidean completeness. In affine differential geometry there are two notions of completeness:

(1) affine completeness, that is, the completeness of the Blaschke metric $G$;

(2) Euclidean completeness, that is, the completeness of the Riemannian metric on $M$ induced from a Euclidean metric on $A^{n+1}$.

Affine Bernstein problem. About complete affine maximal surfaces there are two conjectures, due to Chern and Calabi, both are called an "affine Bernstein problem" (see [CH], $[\mathrm{CA}])$ : 
Chern's CONJECTURE. Let $x_{3}=f\left(x_{1}, x_{2}\right)$ be a strictly convex function defined for all $\left(x_{1}, x_{2}\right) \in A^{2}$. If $M=\left\{\left(x_{1}, x_{2}, f\left(x_{1}, x_{2}\right)\right) \mid\left(x_{1}, x_{2}\right) \in A^{2}\right\}$ is an affine maximal surface, then $M$ must be an elliptic paraboloid.

CALABI's CONJECTURE. A locally strongly convex affine complete surface $x: M \rightarrow A^{3}$ with affine mean curvature $L_{1} \equiv 0$ is an elliptic paraboloid.

These conjectures were generalized to higher dimensions (see [L-S-Z], [CA], [SO-2]). Recently Calabi's conjecture was solved, see [L-J-1], [T-W-2]; Chern's conjecture was solved by Trudinger and Wang X.-J. (see [T-W-1]). In [L-J-2] we proved the following result:

THEOREM 1.6. Let $M$ be a locally strongly convex, Euclidean complete surface in $A^{3}$ with constant affine mean curvature $L_{1}$.

(a) If $L_{1}>0$, then $M$ is an ellipsoid.

(b) If $L_{1}=0$, then $M$ is an elliptic paraboloid.

(c) If $L_{1}<0$, then $M$ has "finite geometry".

As a corollary of Theorem 1.6, we present a new proof of Chern's conjecture about affine maximal surfaces.

Note that the two versions of the affine Bernstein problem assume different completeness conditions. Calabi's conjecture assumes affine completeness; Chern's conjecture assumes Euclidean completeness. Generally, the affine completeness and the Euclidean completeness are not equivalent (see [SCH], [NO]).

\section{Proof of Calabi's Conjecture}

Preliminaries. Let $M$ be a $C^{\infty}$ manifold of dimension 2 and $x: M \rightarrow A^{3}$ a locally strongly convex surface. We choose a local unimodular affine frame field $x, e_{1}, e_{2}, e_{3}$ on $M$ such that

$$
e_{1}, e_{2} \in T_{x} M, \quad \operatorname{det}\left(e_{1}, e_{3}, e_{3}\right)=1, \quad e_{3}=Y .
$$

Denote by $A_{i j}^{k}$ and $B_{i j}$ the local components of the affine Fubini-Pick tensor and the affine Weingarten tensor with respect to the frame field $x, e_{1}, e_{2}, e_{3}$. We have the following local formulas (see [L-S-Z], pp. 52-60); the comma indicates covariant differentiation w.r.t. the Blaschke metric:

$$
\begin{gathered}
x_{, i j}=\sum A_{i j}^{k} e_{k}+G_{i j} Y, \\
U_{, i j}=-\sum A_{i j}^{k} U_{, k}-B_{i j} U, \\
\Delta x=2 Y, \\
\Delta U=-2 L_{1} U, \\
\sum G^{i j} A_{i j k}=0, \\
R=2\left(J+L_{1}\right),
\end{gathered}
$$

where $R, J$ denote the scalar curvature and the Pick invariant, resp. For an affine maximal surface we have $R \geq 0$. 
Again we use isothermal parameters $u, v$. Suppose that $M$ is an affine maximal surface. As above $U$ is harmonic with respect to $u, v$. Let $z:=u+i v$. Define $\alpha$ and $\beta$ by

$$
\alpha:=\frac{1}{2}\left(A_{111}+i A_{222}\right), \quad \beta:=\frac{1}{2}\left(\frac{B_{11}-B_{22}}{2}-i B_{12}\right) .
$$

Let $\|.\|_{G}$ denote the norm with respect to the Blaschke metric. We have (see [L-S-Z], p. 155)

$$
\begin{gathered}
\|\alpha\|^{2}:=\left(\frac{F}{2}\right)^{-3} \cdot \alpha \bar{\alpha}=\frac{1}{2}\|A\|_{G}^{2}, \quad\|\beta\|^{2}:=\left(\frac{F}{2}\right)^{-2} \cdot \beta \bar{\beta}=\frac{1}{2}\|B\|_{G}^{2}, \\
\alpha=i \operatorname{Det}\left(U, U_{z}, U_{z^{2}}\right), \\
\beta=i F^{-1} \operatorname{Det}\left(U_{z}, U_{\bar{z}}, U_{z^{2}}\right), \\
\frac{F}{2}=-i \operatorname{Det}\left(U, U_{z}, U_{\bar{z}}\right) .
\end{gathered}
$$

Calabi calculated the Laplacian of $1 / 2\left(J+\|B\|^{2}\right)=1 / 2 J+\|\beta\|$, he got the following differential inequality (compare [L-S-Z], p. 231):

$$
\Delta\left(\frac{1}{2} J+\|\beta\|\right) \geq 3 J^{2} .
$$

Proof of the conjecture. Using (2.15) we can easily prove the following result of Martinez and Milan (see [M-M] or [L-S-Z], p. 231):

TheOREM 2.1. Let $x: M \rightarrow A^{3}$ be a locally strongly convex affine maximal surface. If $M$ is complete with respect to the Blaschke metric and if there is a constant $N>0$ such that $\|B\|_{G}^{2} \leq N$ everywhere, then $M$ must be an elliptic paraboloid.

Proof. From (2.15) and the assumptions we have

$$
\Delta\left(\frac{1}{2} J+\|\beta\|\right) \geq 3 J^{2} \geq 6\left(\frac{1}{2} J+\|\beta\|\right)^{2}-12\|\beta\|^{2} \geq 6\left(\frac{1}{2} J+\|\beta\|\right)^{2}-6 N .
$$

By a well-known result from geometric analysis it follows that $\frac{1}{2} J+\|\beta\|$ is bounded from above. Being a bounded subharmonic function on a complete 2-dim Riemannian manifold with $R \geq 0, \frac{1}{2} J+\|\beta\|$ must be a constant. It follows that $J=0$ everywhere. Thus $x(M)$ is an elliptic paraboloid.

From the assumption, $x: M \rightarrow A^{3}$ is a locally strongly convex affine maximal surface, which is complete with respect to the Blaschke metric. We want to show that there is a constant $N>0$ such that $\|B\|_{G}^{2} \leq N$ everywhere. We need the following lemma (see [H], p. 635, Lemma 26).

Lemma 1 (Hofer). Let $(X, d)$ be a complete metric space with metric $d$, and $B_{a}(p)=$ $\{x \mid d(p, x) \leq a\}$ be a ball with center $p$ and radius $a$. Let $\Phi$ be a positive continuous function defined on $B_{2 a}(p)$. Then there is a point $q \in B_{a}(p)$ and a positive number $\epsilon \leq a / 2$ such that

$$
\Phi(x) \leq 2 \Phi(q) \text { for all } x \in B_{\epsilon}(q) \quad \text { and } \quad \epsilon \Phi(q) \geq \frac{a}{2} \Phi(p) .
$$

Now we assume that $\|B\|_{G}^{2}$ is not bounded above. Then there is a sequence of points $p_{\ell} \in M$ such that $\|B\|_{G}^{2}\left(p_{\ell}\right) \rightarrow \infty$. We may assume that $M$ is simply connected, otherwise 
we consider its universal covering space. As $M$ is noncompact, complete with $R \geq 0$, it is conformally equivalent to $\mathbb{C}$. Then we may choose global isothermal parameters $u, v$ on $M$ such that the Blaschke metric is given by $G=F\left(d u^{2}+d v^{2}\right)$. Let $B_{1}\left(p_{\ell}\right)$ be the geodesic ball with center $p_{\ell}$ and radius 1 . Consider a family $\Phi(\ell): B_{2}\left(p_{\ell}\right) \rightarrow \mathbb{R}$ of functions, $\ell \in \mathbb{N}$, defined by

$$
\Phi(\ell)=\|\nabla \log F\|_{G}+\|A\|_{G}+\|B\|_{G}^{1 / 2}
$$

In terms of $u, v$ we have

$$
\begin{gathered}
\|\nabla \log F\|_{G}^{2}=\frac{1}{F}\left(\left(\frac{\partial \log F}{\partial u}\right)^{2}+\left(\frac{\partial \log F}{\partial v}\right)^{2}\right) \\
\|A\|_{G}^{2}=\frac{1}{F} \sum\left(A_{i j}^{k}\right)^{2}, \quad\|B\|_{G}^{2}=\frac{1}{F^{2}} \sum\left(B_{i j}\right)^{2} .
\end{gathered}
$$

Using Hofer's Lemma we find a sequence of points $q_{\ell}$ and positive numbers $\epsilon_{\ell}$ such that

$$
\begin{gathered}
\Phi(x) \leq 2 \Phi\left(q_{\ell}\right) \quad \forall x \in B_{\epsilon_{\ell}}\left(q_{\ell}\right) \\
\epsilon_{\ell} \Phi\left(q_{\ell}\right) \geq \frac{1}{2} \Phi\left(p_{\ell}\right) \rightarrow \infty .
\end{gathered}
$$

The restriction of the surface $x$ to the balls $B_{\epsilon_{\ell}}\left(q_{\ell}\right)$ defines a family $M(\ell)$ of maximal surfaces. For every $\ell$, we normalize $M(\ell)$ as follows:

STEP 1. Denote by $u(\ell), v(\ell)$ the restriction of the isothermal parameters of $M$ to $M(\ell)$. First we take a parameter transformation on $M(\ell)$ :

$$
\hat{u}(\ell)=c(\ell) u(\ell), \quad \hat{v}(\ell)=c(\ell) v(\ell), \quad c(\ell)>0,
$$

where $c(\ell)$ is a constant. Choosing $c(\ell)$ appropriately and using an obvious notation $\hat{F}$, we may assume that, for every $\ell$, we have $\hat{F}\left(q_{\ell}\right)=1$. Note that, under the parameter transformation (2.18), $\Phi$ is invariant.

STEP 2. We use the Weierstrass representation for affine maximal surfaces (see Section 1) to define, for every $\ell$, a new surface $\tilde{M}(\ell)$ from $M(\ell)$ via its conormal by

$$
\tilde{U}(\ell)=\lambda(\ell) U(\ell), \quad \lambda(\ell)>0 ;
$$

we introduce new parameters $\tilde{u}(\ell), \tilde{v}(\ell)$ by

$$
\tilde{u}(\ell)=b(\ell) \hat{u}(\ell), \quad \tilde{v}(\ell)=b(\ell) \hat{v}(\ell), \quad b(\ell)>0,
$$

where $\lambda(\ell)$ and $b(\ell)$ are appropriate constants. From the foregoing conormal equation one easily verifies that each $\tilde{M}(\ell)$ again is a locally strongly convex maximal surface. We now choose $\lambda(\ell)=(b(\ell))^{2 / 3}, b(\ell)=\Phi\left(q_{\ell}\right)$. Using again an obvious notation $\tilde{F}$, $\tilde{\Phi}$, from (2.11)-(2.14) one can see that

$$
\tilde{F}=\hat{F}, \quad \tilde{\Phi}(\ell)=\frac{1}{b(\ell)} \Phi(\ell) .
$$

The first equation is trivial. We calculate the second one. We can easily get

$$
\|\nabla \log \tilde{F}\|_{\tilde{G}}=\frac{1}{b}\|\nabla \log \hat{F}\|_{G}
$$

From (2.11)-(2.14) and our choice $\lambda^{3}=b^{2}$ we have

$$
\|\tilde{B}\|_{\tilde{G}}^{2}=2\|\tilde{\beta}\|^{2}=2 \frac{1}{b^{4}}\|\beta\|^{2}=\frac{1}{b^{4}}\|B\|_{G}^{2},
$$




$$
\|\tilde{A}\|_{\tilde{G}}^{2}=2\|\tilde{\alpha}\|^{2}=2 \frac{\lambda^{6}}{b^{6}}\|\alpha\|^{2}=2 \frac{1}{b^{2}}\|\alpha\|^{2}=\frac{1}{b^{2}}\|A\|_{G}^{2} .
$$

Then the second equality follows.

We denote $\tilde{B}_{a}\left(q_{\ell}\right)=\left\{x \in \tilde{M}(\ell) \mid \tilde{d}(\ell)\left(x, q_{\ell}\right) \leq a\right\}$, where $\tilde{d}(\ell)$ is the geodesic distance function with respect to the Blaschke metric on $\tilde{M}(\ell)$. Then $\tilde{\Phi}(\ell)$ is defined on the geodesic ball $\tilde{B}_{r(\ell)}\left(q_{\ell}\right)$ with $r(\ell)=\epsilon_{\ell} \Phi\left(q_{\ell}\right) \geq \frac{1}{2} \Phi\left(p_{\ell}\right) \rightarrow \infty$. From $(2.16)$ we have

$$
\begin{gathered}
\tilde{\Phi}\left(q_{\ell}\right)=1, \\
\tilde{\Phi}(x) \leq 2, \quad \forall x \in \tilde{B}_{r(\ell)}\left(q_{\ell}\right) .
\end{gathered}
$$

STEP 3. For any $\ell$ we introduce new parameters $\xi_{1}(\ell), \xi_{2}(\ell)$ as follows:

$$
\xi_{1}(\ell)=\tilde{u}(\ell)-\tilde{u}(\ell)\left(q_{\ell}\right), \quad \xi_{2}(\ell)=\tilde{v}(\ell)-\tilde{v}(\ell)\left(q_{\ell}\right) .
$$

Then at $q_{\ell},\left(\xi_{1}, \xi_{2}\right)=(0,0)$ for any $\ell$, and we can identify the parametrization $\left(\xi_{1}, \xi_{2}\right)$ for any index $\ell$. Let $\tilde{x}(\ell)$ denote the position vector of $\tilde{M}(\ell)$. An appropriate unimodular affine transformation gives

$$
\begin{gathered}
\tilde{x}(\ell)(o)=(0,0,0), \\
\tilde{x}_{\xi_{1}}(\ell)(o)=e_{1}=(1,0,0), \\
\tilde{x}_{\xi_{2}}(\ell)(o)=e_{2}=(0,1,0), \\
\tilde{Y}(\ell)(o)=(0,0,1) .
\end{gathered}
$$

Consider the open geodesic balls

$$
\Omega(\ell):=\left\{\left(\xi_{1}, \xi_{2}\right) \in \mathbb{R}^{2} \mid \tilde{d}(\ell)(o, \xi)<r(\ell)\right\}
$$

and the sequence $\tilde{M}(\ell)$ of maximal surfaces $\tilde{x}(\ell): \Omega(\ell) \rightarrow A^{3}$. They satisfy $(2.21)-(2.24)$ and the conditions

$$
\begin{gathered}
\tilde{F}(\ell)(o)=1, \\
\tilde{\Phi}(\ell)(o)=1, \quad \tilde{\Phi}(\ell)(\xi) \leq 2 \quad \forall \xi \in \Omega(\ell), \\
r(\ell) \rightarrow \infty .
\end{gathered}
$$

It follows from (2.7)-(2.10) and (2.22)-(2.25) that, for any $\ell,\left(\tilde{U}_{\xi_{1}}, \tilde{U}_{\xi_{2}}, \tilde{U}\right)(0)=I$, where $I$ is the unit matrix. We need the following lemma

LEMma 2. Let $M$ be an affine maximal surface defined in a neighborhood of $0 \in \mathbb{R}^{2}$. Suppose that, with the notations from above,

$$
F(o)=1, \quad\left(U_{\xi_{1}}, U_{\xi_{2}}, U\right)(o)=I,
$$

(ii) $\left(\frac{1}{F} \sum\left(\frac{\partial(\log F)}{\partial \xi_{i}}\right)^{2}\right)^{1 / 2}+\left(\frac{1}{F} \sum\left(A_{i j}^{k}\right)^{2}\right)^{1 / 2}+\left(\frac{1}{F}\left(\sum\left(B_{i j}\right)^{2}\right)^{1 / 2}\right)^{1 / 2} \leq 2$.

Denote $D:=\left\{\left(\xi_{1}, \xi_{2}\right) \mid \xi_{1}^{2}+\xi_{2}^{2} \leq \frac{1}{2}\right\}$. Then there is a constant $C_{1}>0$ such that, for $\left(\xi_{1}, \xi_{2}\right) \in D$, the following estimates hold:

(1)

$$
\frac{4}{9} \leq F \leq 4
$$


(2)

$$
|U|+\left|U_{\xi_{1}}\right|+\left|U_{\xi_{2}}\right| \leq C_{1}
$$

where $|\cdot|$ denotes the canonical norm in $\mathbb{R}^{3}$;

(3) denote $r_{o}=\frac{1}{3}$; then $\bar{\Omega}_{r_{o}} \subset\left\{\xi_{1}^{2}+\xi_{2}^{2}<\frac{1}{4}\right\} \subset D$, where $\Omega_{r_{o}}$ is the geodesic ball with center $o$ and radius $r_{o}$ with respect to the Blaschke metric.

Proof of Lemma 2. (1) Consider an arbitrary curve $\Gamma=\left\{\xi_{1}=a_{1} s, \xi_{2}=a_{2} s, a_{1}^{2}+a_{2}^{2}=\right.$ $1, s \geq 0\}$. By assumption we have

$$
\frac{1}{F}\left(\frac{\partial \log F}{\partial s}\right)^{2} \leq 2, \quad F(0)=1 .
$$

Solving this differential inequality with $F(0)=1$, we get

$$
\left(\frac{1}{1+\frac{\sqrt{2}}{2} s}\right)^{2} \leq F(s) \leq\left(\frac{1}{1-\frac{\sqrt{2}}{2} s}\right)^{2} .
$$

From the assumption we have $s \leq \frac{1}{\sqrt{2}}$, then (1) follows.

(2) Note that the Christoffel symbols are given by $\partial \log F / \partial \xi_{i}$. Along the curve $\Gamma$ the structure equation $U_{, i j}=-\sum A_{i j}^{k} U_{, k}-B_{i j} U$ gives an ODE which can be written in matrix form:

$$
\frac{d X}{d s}=X H
$$

where $X=\left(U_{\xi_{1}}, U_{\xi_{2}}, U\right)$, and $H$ is a matrix, whose elements depend on $B_{i j}, A_{i j}^{k}$ and $\partial \log F / \partial \xi_{i}$. It follows from (2.27) that

$$
\frac{d X^{t}}{d s}=H^{t} X^{t}
$$

where we use an obvious notation for the transpose of a matrix. Then

$$
\frac{d\left(X^{t} X\right)}{d s}=H^{t} X^{t} X+X^{t} X H
$$

Denote $f=\operatorname{Tr}\left(X^{t} X\right)$. Taking the trace of (2.30) we get

$$
\frac{d f}{d s}=\operatorname{Tr}\left(H^{t} X^{t} X\right)+\operatorname{Tr}\left(X^{t} X H\right) \leq C f,
$$

where $C$ is a constant. Deriving the last inequality we used (1) and the condition (ii). Solving (2.30) with the condition (i) we get (2). From (1) we immediately get (3).

We continue with the proof of the conjecture. Since $r(\ell) \rightarrow \infty$, we have $D \subset \Omega(\ell)$ for $\ell$ big enough. In fact, by (1), the geodesic distance from 0 to the boundary of $D$ with respect to the Blaschke metric on $\tilde{M}(\ell)$ is less than $\sqrt{2}$. Using (2) and a standard elliptic estimate we get a $C^{k}$-estimate, independent of $\ell$, for any $k$. It follows that there is a ball $\left\{\xi_{1}^{2}+\xi_{2}^{2} \leq C_{2}\right\}$ and a subsequence (still indexed by $\ell$ ) such that $\tilde{U}(\ell)$ converges to $\tilde{U}$ on the ball, and correspondingly all derivatives, where $C_{2}<\frac{1}{2}$ is very close to $\frac{1}{2}$. Thus, as limit, we get a maximal surface $\tilde{M}$, defined on the ball, which contains a geodesic ball $\bar{\Omega}_{r_{o}}$. We now extend the surface $\tilde{M}$ as follows: For every boundary point $p=\left(\xi_{1 o}, \xi_{2 o}\right)$ of the geodesic ball $\bar{\Omega}_{r_{o}}$ we first make the parameter transformation: $\tilde{\xi}_{i}=b\left(\xi_{i}-\xi_{i o}\right)$ such 
that at $p,\left(\tilde{\xi}_{1}, \tilde{\xi}_{2}\right)=(0,0)$, and for the limit surface $\tilde{M}$ we have $\tilde{F}(p)=1$. We choose frame $e_{1}, e_{2}, e_{3}$ at $p$ such that $e_{1}=\tilde{x}_{\tilde{\xi}_{1}}, e_{2}=\tilde{x}_{\tilde{\xi}_{2}}, e_{3}=\tilde{Y}$. We have

$$
\tilde{F}(\ell)(p) \rightarrow \tilde{F}(p)=1, \quad\left(\tilde{U}_{\tilde{\xi}_{1}}(\ell), \tilde{U}_{\tilde{\xi}_{2}}(\ell), \tilde{U}(\ell)\right)(p) \rightarrow I \quad \text { as } \ell \rightarrow \infty .
$$

It is easy to see that under the conditions ( $\mathrm{i}^{\prime}$ ) and (ii) in Lemma 2, the estimates (1), (2) and (3) in Lemma 2 remain true. By the same argument as above we conclude that there is a ball around $p$ and a subsequence $\ell_{k}$, such that $\tilde{U}\left(\ell_{k}\right)$ converges to $\tilde{U}^{\prime}$ on the ball, and correspondingly all derivatives. As limit, we get a maximal surface $\tilde{M}^{\prime}$, which contains a geodesic ball of radius $r_{o}$ around $p$. Then we return to the original parameters $\xi_{1}, \xi_{2}$ and the original frame $e_{1}, e_{2}, e_{3}$ at 0 . Note that the geodesic distance is independent of the choice of the parameters and the frames. It is obvious that $\tilde{M}$ and $\tilde{M}^{\prime}$ agree on the common part. We repeat this procedure to extend $\tilde{M}$ to be defined on $\bar{\Omega}_{2 r_{o}}$, etc. In this way we may extend $\tilde{M}$ to be an affine complete maximal surface defined in a domain $\Omega \subset \mathbb{R}^{2}$; using (24) and (25) we get

$$
\|\tilde{B}\|_{\tilde{G}} \leq 2, \quad \tilde{\Phi}(0)=1 .
$$

By Theorem 1, $\tilde{M}$ must be an elliptic paraboloid, given by

$$
x_{3}=\frac{1}{2}\left(x_{1}^{2}+x_{2}^{2}\right)
$$

where $x_{1}, x_{2}, x_{3}$ are the coordinates in $A^{3}$ with respect to the frame $e_{1}, e_{2}, e_{3}$. For a paraboloid we have $\|\tilde{A}\|_{\tilde{G}}=0,\|\tilde{B}\|_{\tilde{G}}^{2}=0, \tilde{R}=0$ identically, and $\tilde{G}=d x_{1}^{2}+d x_{2}^{2}$. Thus

$$
\|\nabla \log \tilde{F}\|_{\tilde{G}}(0)=1 \text {. }
$$

We consider $\log \tilde{F}$ as a function of $x_{1}, x_{2}$. Since the scalar curvature vanishes identically, $\tilde{R}=0$, from the formula

$$
\Delta \log \tilde{F}=-\tilde{R}
$$

we conclude that $\log \tilde{F}$ is a harmonic function. As $\|\nabla \log \tilde{F}\|_{\tilde{G}} \leq 2, \log \tilde{F}$ must be a linear function. In view of (2.32), without loss of generality, we may assume that $\log \tilde{F}=x_{1}$. We introduce complex coordinates and write $w=\xi_{1}+i \xi_{2}, z=x_{1}+i x_{2}$, then $w(z)$ is a holomorphic or anti-holomorphic function. We consider the case that $w$ is holomorphic. For the case that $w$ is anti-holomorphic, the discussion is similar. Since $\tilde{G}=|d z|^{2}=$ $\tilde{F}|d w|^{2}$ we have $\left|w^{\prime}\right|^{2}=\tilde{F}^{-1}=e^{-x_{1}}$. Let $Q=e^{\frac{z}{2}}$. Then $\left|w^{\prime} Q\right|=1$. From the maximum principle we get $w^{\prime} Q=C$ where $C$ is a constant with $|C|=1$. So $w^{\prime}=C e^{-\frac{z}{2}}$. It follows that $w=-2 C e^{-\frac{z}{2}}+E$, where $E$ is a constant. Since $e^{-\frac{z}{2}}$ has period $2 \pi$ for $x_{2}$, we have a covering map $A^{2} \rightarrow \Omega$; this is impossible. We get a contradiction. So $\|B\|_{G}$ must be bounded above on $M$. By Theorem $1 M$ is an elliptic paraboloid. We have proved the following

TheOREM 2.2. Let $x: M \rightarrow A^{3}$ be a locally strongly convex affine maximal surface. If $M$ is complete with respect to the Blaschke metric, then $M$ must be an elliptic paraboloid.

\section{Euclidean complete affine surfaces with constant affine mean curvature}

Introduction. The classification of locally strongly convex, affine-complete affine hyperspheres had attracted many geometers during the last decades. For the history of this problem and the contributions of different authors we refer to the monograph [L-S-Z], 
pp. 84-85. Obviously, every affine hypersphere has constant affine mean curvature $L_{1}$. Thus an interesting and important problem is the classification of locally strongly convex, complete affine hypersurfaces with constant affine mean curvature $L_{1}$. We shall show that the study of locally strongly convex, Euclidean complete hypersurfaces with constant affine mean curvature $L_{1}=L$ is equivalent to the study of the convex solutions of the fourth order P.D.E.

$$
\Delta\left[\operatorname{det}\left(\frac{\partial^{2} f}{\partial x_{i} \partial x_{j}}\right)\right]^{-1 /(n+2)}=-n L\left[\operatorname{det}\left(\frac{\partial^{2} f}{\partial x_{i} \partial x_{j}}\right)\right]^{-1 /(n+2)} .
$$

In this paper, we consider the convex solutions of the equation $(*)$ for $n=2$. We shall prove that a) if $L>0$, then there is no convex solution of $(*)$ which is defined for all $\left.\left(x_{1}, x_{2}\right) \in \mathbb{R}^{2} ; \mathrm{b}\right)$ if $L=0$ and $f\left(x_{1}, x_{2}\right)$ is a convex solution of $(*)$, which is defined for all $\left(x_{1}, x_{2}\right) \in \mathbb{R}^{2}$, then $f\left(x_{1}, x_{2}\right)$ must be a quadratic polynomial. In the language of affine differential geometry, our main theorems can be stated as follows:

THEOREM 3.1. Every locally strongly convex, Euclidean complete surface with constant affine mean curvature is affine complete.

To state our Theorem 3.2 we introduce a terminology. A locally strongly convex hypersurface is said to have "finite geometry" if $\|B\|_{k}$ and $\|A\|_{k}$ are bounded, where

$$
\begin{aligned}
\|B\|_{k} & :=\|B\|+\|\nabla B\|+\left\|\nabla^{2} B\right\|+\cdots+\left\|\nabla^{k} B\right\|, \\
\|A\|_{k} & :=\|A\|+\|\nabla A\|+\left\|\nabla^{2} A\right\|+\cdots+\left\|\nabla^{k} A\right\| .
\end{aligned}
$$

Using Theorem 3.1 we immediately get the following result:

THEOREM 3.2. Let $M$ be a locally strongly convex, Euclidean complete surface in $A^{3}$ with constant affine mean curvature $L_{1}$.

(a) If $L_{1}>0$, then $M$ is an ellipsoid.

(b) If $L_{1}=0$, then $M$ is an elliptic paraboloid.

(c) If $L_{1}<0$, then $M$ has "finite geometry".

As a corollary of Theorem 3.2, we present a new proof of Chern's conjecture about affine maximal surfaces.

Fundamental formulas. Consider a locally strongly convex hypersurface $x: M \rightarrow A^{n+1}$, which is given as a graph by a strictly convex function

$$
x_{n+1}=f\left(x_{1}, \ldots, x_{n}\right) .
$$

Then, the Blaschke metric $G$ is given as in Section 1 and the affine conormal vector field $U$ can be identified with

$$
U=\left[\operatorname{det}\left(\frac{\partial^{2} f}{\partial x_{i} \partial x_{j}}\right)\right]^{-1 /(n+2)}\left(-\frac{\partial f}{\partial x_{1}}, \ldots,-\frac{\partial f}{\partial x_{n}}, 1\right) .
$$

The formula $\Delta U=-n L_{1} U$ implies that $x(M)$ is a locally strongly convex hypersurface with constant affine mean curvature $L_{1} \equiv L$ if and only if $f$ satisfies the following P.D.E.:

$$
\Delta\left[\operatorname{det}\left(\frac{\partial^{2} f}{\partial x_{i} \partial x_{j}}\right)\right]^{-1 /(n+2)}=-n L\left[\operatorname{det}\left(\frac{\partial^{2} f}{\partial x_{i} \partial x_{j}}\right)\right]^{-1 /(n+2)} .
$$


Denote

$$
\rho:=\left[\operatorname{det}\left(\frac{\partial^{2} f}{\partial x_{i} \partial x_{j}}\right)\right]^{-1 /(n+2)}
$$

Then (3.1) gives

$$
\Delta \rho=-n L_{1} \rho .
$$

Note that in terms of $x_{1}, \ldots, x_{n}$ we have $\left(\operatorname{det}\left(G_{k l}\right)\right)^{1 / 2}=\frac{1}{\rho}$. By a direct calculation we get

$$
\Delta=\frac{1}{\rho} \sum f^{i j} \frac{\partial^{2}}{\partial x_{i} \partial x_{j}}-\frac{2}{\rho^{2}} \sum f^{i j} \frac{\partial \rho}{\partial x_{j}} \frac{\partial}{\partial x_{i}}+\frac{1}{\rho} \sum \frac{\partial f^{i j}}{\partial x_{i}} \frac{\partial}{\partial x_{j}}
$$

where $\left(f^{i j}\right)$ denotes the inverse matrix of $\left(f_{i j}\right)$ and $f_{i j}=\partial^{2} f / \partial x_{i} \partial x_{j}$. Differentiate the equality $\sum f^{i k} f_{k j}=\delta_{j}^{i}$; one finds

$$
\sum \frac{\partial f^{i k}}{\partial x_{i}} f_{k j}=-\sum f^{i k} \frac{\partial f_{k j}}{\partial x_{i}}=\frac{(n+2)}{\rho} \frac{\partial \rho}{\partial x_{j}} .
$$

It follows that

$$
\sum \frac{\partial f^{i k}}{\partial x_{i}}=\frac{(n+2)}{\rho} \sum f^{j k} \frac{\partial \rho}{\partial x_{j}} .
$$

Inserting (3.4) into (3.3) we obtain

$$
\Delta=\frac{1}{\rho} \sum f^{i j} \frac{\partial^{2}}{\partial x_{i} \partial x_{j}}+\frac{n}{\rho^{2}} \sum f^{i j} \frac{\partial \rho}{\partial x_{j}} \frac{\partial}{\partial x_{i}} .
$$

To find the affine normal $\mathrm{Y}$ and calculate the affine Weingarten tensor $B_{i j}$, we let (see $[\mathrm{CH}])$

$$
e_{i}^{*}=e_{i}, \quad 1 \leq i \leq n, \quad e_{n+1}^{*}=e_{n+1}+\sum a_{n+1}^{i} e_{i},
$$

where $e_{n+1}^{*}$ is in the affine normal direction. Then the coefficients $a_{n+1}^{i}$ are determined by

It follows that

$$
\sum a_{n+1}^{j} f_{j i}=\frac{\partial}{\partial x_{i}} \log \rho .
$$

and hence

$$
a_{n+1}^{i}=\sum f^{j i} \frac{\partial}{\partial x_{j}} \log \rho,
$$

Therefore

$$
e_{n+1}^{*}=e_{n+1}+\sum f^{j i} \frac{\partial}{\partial x_{j}} \log \rho \cdot e_{i}
$$

$$
Y=H^{1 /(n+2)} e_{n+1}^{*}=H^{1 /(n+2)} \sum f^{j i} \frac{\partial}{\partial x_{j}} \log \rho \cdot e_{i}+H^{1 /(n+2)} e_{n+1},
$$

where $H=\operatorname{det}\left(\partial^{2} f / \partial x_{i} \partial x_{j}\right)$.

Let $x$ be the position vector of the hypersurface $M$. We can write

$$
d x=\sum w^{\alpha} e_{\alpha}^{*}, \quad d e_{\alpha}^{*}=\sum w_{\alpha}^{\beta} e_{\beta}^{*} .
$$

$w^{\alpha}, w_{\alpha}^{\beta}$ denote the Maurer-Cartan forms of the unimodular affine group. We compute $w_{n+1}^{i}=d a_{n+1}^{i}-a_{n+1}^{i} d \log \rho=\sum\left(\frac{\partial}{\partial x_{j}}\left(f^{k i} \frac{\partial}{\partial x_{k}} \log \rho\right)-f^{k i} \frac{\partial}{\partial x_{k}} \log \rho \cdot \frac{\partial}{\partial x_{j}} \log \rho\right) w^{j}$. 
Therefore the affine Weingarten tensor is

$$
\begin{aligned}
B_{i j} & =\sum\left(-\frac{\partial}{\partial x_{i}}\left(f^{l k} \frac{\partial}{\partial x_{l}} \log \rho\right)+f^{l k} \frac{\partial}{\partial x_{l}} \log \rho \frac{\partial}{\partial x_{i}} \log \rho\right) f_{k j} \\
& =-\frac{1}{\rho} \frac{\partial^{2} \rho}{\partial x_{i} \partial x_{j}}+\frac{2}{\rho^{2}} \frac{\partial \rho}{\partial x_{i}} \frac{\partial \rho}{\partial x_{j}}+\sum \frac{f^{k l}}{\rho} \frac{\partial \rho}{\partial x_{l}} \frac{\partial f_{i j}}{\partial x_{k}} .
\end{aligned}
$$

Consider the Legendre transformation relative to $f$ :

$$
\begin{gathered}
\xi_{i}=\frac{\partial f}{\partial x_{i}}\left(x_{1}, \ldots, x_{n}\right), \\
u\left(\xi_{1}, \ldots, \xi_{n}\right)=\sum x_{i} \frac{\partial f}{\partial x_{i}}\left(x_{1}, \ldots, x_{n}\right)-f\left(x_{1}, \ldots, x_{n}\right),
\end{gathered}
$$

and denote by $\Omega^{*}$ the Legendre transformation domain of $f$, i.e. $u: \Omega^{*} \rightarrow \mathbb{R}$ and

$$
\Omega^{*}=\left\{\left(\xi_{1}(x), \ldots, \xi_{n}(x)\right) \mid x \in \Omega\right\} .
$$

In terms of the coordinates $\left(\xi_{1}, \ldots, \xi_{n}\right)$ the Blaschke metric is given by

$$
G_{i j}=\rho \frac{\partial^{2} u}{\partial \xi_{i} \partial \xi_{j}}
$$

and $\left(\partial^{2} u / \partial \xi_{i} \partial \xi_{j}\right)$ is the inverse matrix of $\left(\partial^{2} f / \partial x_{i} \partial x_{j}\right)$. We have

$$
\begin{gathered}
\rho=\left[\operatorname{det}\left(\frac{\partial^{2} u}{\partial \xi_{i} \partial \xi_{j}}\right)\right]^{1 /(n+2)}, \\
\sqrt{\operatorname{det}\left(G_{k l}\right)}=\rho^{n+1} \\
\Delta=\frac{1}{\sqrt{\operatorname{det}\left(G_{k l}\right)}} \sum \frac{\partial}{\partial \xi_{i}}\left(G^{i j} \sqrt{\operatorname{det}\left(G_{k l}\right)} \frac{\partial}{\partial \xi_{j}}\right)
\end{gathered}
$$

(see [L-S-Z], p. 91). By a similar calculation as above we get

$$
\Delta=\frac{1}{\rho} \sum u^{i j} \frac{\partial^{2}}{\partial \xi_{i} \partial \xi_{j}}-\frac{2}{\rho^{2}} \sum u^{i j} \frac{\partial \rho}{\partial \xi_{j}} \frac{\partial}{\partial \xi_{i}} .
$$

Estimates for the function $\rho$. In this section we give some estimates which play an important role in our proof. Suppose that $f\left(x_{1}, x_{2}\right)$ is a strictly convex function defined in a bounded convex domain $\Omega$ such that the graph

$$
M=\left\{\left(x_{1}, x_{2}, f\left(x_{1}, x_{2}\right)\right) \mid\left(x_{1}, x_{2}\right) \in \Omega\right\}
$$

is a locally strongly convex surface with constant affine mean curvature $L_{1}$. As above consider the Legendre transformation relative to $f$ with $u: \Omega^{*} \rightarrow \mathbb{R}$. Then $u$ is a convex function defined in $\Omega^{*}$ and $M$ can be represented in terms of $\xi_{1}, \xi_{2}$ as follows:

$$
x=\left(x_{1}, x_{2}, f\left(x_{1}, x_{2}\right)\right)=\left(\frac{\partial u}{\partial \xi_{1}}, \frac{\partial u}{\partial \xi_{2}},-u+\sum \xi_{i} \frac{\partial u}{\partial \xi_{i}}\right) .
$$

First we estimate $\rho=\left[\operatorname{det}\left(\partial^{2} u / \partial \xi_{i} \partial \xi_{j}\right)\right]^{1 / 4}$ from above. For any $\xi_{0}=\left(\xi_{1}^{0}, \xi_{2}^{0}\right) \in \Omega^{*}$, we choose $r>0$ such that $0<r<\operatorname{dist}\left(\xi_{0}, \partial \Omega^{*}\right)$. We introduce the notations

$$
\begin{gathered}
\overline{B_{r}\left(\xi_{0}\right)}=\left\{\left(\xi_{1}, \xi_{2}\right) \mid \sum\left(\xi_{i}-\xi_{i}^{\circ}\right)^{2} \leq r^{2}\right\}, \quad h=\sum\left(\xi_{i}-\xi_{i}^{\circ}\right)^{2}, \\
g=\sum\left(\frac{\partial u}{\partial \xi_{i}}\right)^{2}, \quad A=\frac{\max }{B_{r}\left(\xi_{0}\right)} g .
\end{gathered}
$$


Consider the function

$$
F=\exp \left\{-\frac{m}{r^{2}-h}+\frac{1}{\alpha A} g\right\} \rho
$$

defined on $\overline{B_{r}\left(\xi_{0}\right)}$, where $m$ and $\alpha$ are appropriate positive constants to be determined later. Clearly, $F$ attains its supremum at some interior point $\xi^{*}$ of $\overline{B_{r}\left(\xi_{0}\right)}$. We choose a local orthonormal frame field $e_{1}, e_{2}$ of the Blaschke metric on $M$ near

$$
p^{*}=\left(\frac{\partial u}{\partial \xi_{1}}\left(\xi^{*}\right), \frac{\partial u}{\partial \xi_{2}}\left(\xi^{*}\right),-u\left(\xi^{*}\right)+\sum \xi_{i}^{*} \frac{\partial u}{\partial \xi_{i}}\left(\xi^{*}\right)\right) .
$$

Then, at $p^{*}$,

$$
\begin{gathered}
F_{, i}=0, \\
\sum F_{, i i} \leq 0 .
\end{gathered}
$$

We calculate both expressions explicitly

$$
\begin{gathered}
-\frac{m}{\left(r^{2}-h\right)^{2}} h_{, i}+\frac{1}{\alpha A} g_{, i}+\frac{\rho_{, i}}{\rho}=0 \\
-\frac{2 m}{\left(r^{2}-h\right)^{3}} \sum h_{, i}^{2}-\frac{m}{\left(r^{2}-h\right)^{2}} \Delta h+\frac{1}{\alpha A} \Delta g-\frac{\sum \rho_{, i}^{2}}{\rho^{2}}+\frac{\Delta \rho}{\rho} \leq 0 .
\end{gathered}
$$

Using the formulas (3.5) (3.8) and (3.2), we get

$$
\begin{gathered}
-\frac{2 m}{\left(r^{2}-h\right)^{3}} \sum h_{, i}^{2}-\frac{2 m}{\left(r^{2}-h\right)^{2}} \frac{1}{\rho} \sum u^{i i}+\frac{2 m}{\left(r^{2}-h\right)^{2}} \sum \frac{\rho_{, i}}{\rho} h_{, i} \\
+\frac{2}{\alpha A} \frac{1}{\rho} \sum f^{i i}+\frac{2}{\alpha A} \sum \frac{\rho_{, i}}{\rho} g_{, i}-\sum \frac{\rho_{, i}^{2}}{\rho^{2}}-2\left|L_{1}\right| \leq 0 .
\end{gathered}
$$

Inserting (3.12) into (3.14) and applying the Cauchy-Schwarz inequality, we obtain

$$
\begin{gathered}
-\frac{2 m}{\left(r^{2}-h\right)^{3}} \sum h_{, i}^{2}-\frac{2 m}{\left(r^{2}-h\right)^{2}} \frac{1}{\rho} \sum u^{i i}+\frac{1}{2} \frac{m^{2}}{\left(r^{2}-h\right)^{4}} \sum h_{, i}^{2} \\
+\frac{2}{\alpha A} \frac{1}{\rho} \sum f^{i i}-\frac{5}{(\alpha A)^{2}} \sum g_{, i}^{2}-2\left|L_{1}\right| \leq 0 .
\end{gathered}
$$

Note that

$$
\begin{aligned}
\sum g_{, i}^{2} & =\sum \frac{u^{i j}}{\rho} \frac{\partial g}{\partial \xi_{i}} \frac{\partial g}{\partial \xi_{j}}=\frac{4}{\rho} \sum u^{i j} u_{k} u_{k i} u_{l} u_{l j} \\
& =\frac{4}{\rho} \sum u_{k l} u_{k} u_{l} \leq \frac{4}{\rho} \sum u_{i i} \cdot \sum u_{i}^{2} .
\end{aligned}
$$

Inserting (3.16) into (3.15), we get

$$
\begin{gathered}
-\frac{2 m}{\left(r^{2}-h\right)^{3}} \sum h_{, i}^{2}-\frac{2 m}{\left(r^{2}-h\right)^{2}} \frac{1}{\rho} \sum u^{i i}+\frac{1}{2} \frac{m^{2}}{\left(r^{2}-h\right)^{4}} \sum h_{, i}^{2} \\
+\frac{2}{\alpha A} \frac{1}{\rho} \sum f^{i i}-\frac{20}{\alpha^{2} A} \frac{1}{\rho} \sum u_{i i}-2\left|L_{1}\right| \leq 0 .
\end{gathered}
$$

We choose $m=4 r^{2}$ and $\alpha=20$. Then

$$
\frac{1}{20 A} \sum u_{i i} \leq \frac{2 m}{\left(r^{2}-h\right)^{2}} \sum u^{i i}+2\left|L_{1}\right| \rho .
$$


Denote by $\lambda_{1}, \lambda_{2}$ the eigenvalues of $\left(\partial^{2} u / \partial \xi_{i} \partial \xi_{j}\right)=\left(u_{i j}\right)$. Recall that $\rho=$ $\left[\operatorname{det}\left(\partial^{2} u / \partial \xi_{i} \partial \xi_{j}\right)\right]^{\frac{1}{4}}=\left(\lambda_{1} \lambda_{2}\right)^{\frac{1}{4}}$. It follows from (3.18) that

$$
\begin{aligned}
\frac{1}{20 A}\left(\lambda_{1}+\lambda_{2}\right) & \leq \frac{2 m}{\left(r^{2}-h\right)^{2}}\left(\frac{1}{\lambda_{1}}+\frac{1}{\lambda_{2}}\right)+2\left|L_{1}\right|\left(\lambda_{1} \lambda_{2}\right)^{\frac{1}{4}} \\
& =\frac{2 m}{\left(r^{2}-h\right)^{2}} \frac{\lambda_{1}+\lambda_{2}}{\lambda_{1} \lambda_{2}}+2\left|L_{1}\right|\left(\lambda_{1} \lambda_{2}\right)^{\frac{1}{4}}
\end{aligned}
$$

and hence

$$
\frac{1}{20 A} \lambda_{1} \lambda_{2} \leq \frac{2 m}{\left(r^{2}-h\right)^{2}}+2\left|L_{1}\right| \frac{\left(\lambda_{1} \lambda_{2}\right)^{\frac{1}{2}}\left(\lambda_{1} \lambda_{2}\right)^{\frac{3}{4}}}{\lambda_{1}+\lambda_{2}} \leq \frac{2 m}{\left(r^{2}-h\right)^{2}}+\left|L_{1}\right|\left(\lambda_{1} \lambda_{2}\right)^{\frac{3}{4}},
$$

i.e.

$$
\rho^{4}-20 A\left|L_{1}\right| \rho^{3}-40 A \frac{m}{\left(r^{2}-h\right)^{2}} \leq 0 .
$$

Consequently, from the Cauchy-Schwarz inequality, we have

$$
\rho^{4}-400 A^{2}\left|L_{1}\right|^{2} \rho^{2}-80 A \frac{m}{\left(r^{2}-h\right)^{2}} \leq 0 .
$$

The left hand side is a quadratic expression in $\rho^{2}$. If one consider its zeroes, it follows that

$$
\rho^{2} \leq 400 A^{2}\left|L_{1}\right|^{2}+\left(80 A \frac{m}{\left(r^{2}-h\right)^{2}}\right)^{\frac{1}{2}} .
$$

Therefore

$$
\rho \leq(800)^{1 / 2} A\left|L_{1}\right|+(320)^{\frac{1}{4}} A^{1 / 4} \frac{m^{1 / 4}}{\left(r^{2}-h\right)^{1 / 2}} .
$$

With our special choice of $m$ and $\alpha$, from (3.9) we thus get

$$
F \leq \exp \left\{\frac{1}{20}\right\}\left[(800)^{1 / 2} A\left|L_{1}\right|+(20)^{1 / 4} \exp \{-1 / 2\} \frac{A^{1 / 4}}{r^{1 / 2}}\right],
$$

which holds at $\xi^{*}$, where $F$ attains its supremum. Hence, at any point of $B_{\frac{1}{2} r}\left(\xi_{0}\right)$, we have

$$
\rho \leq \exp \left\{\frac{161}{20}\right\}\left[(800)^{1 / 2} A\left|L_{1}\right|+(20)^{1 / 4} \exp \{-1 / 2\} \frac{A^{1 / 4}}{r^{1 / 2}}\right] .
$$

Using (3.20), we obtain the following lemma:

LEMma 3.3. Let $\Omega$ be a bounded convex domain with $(0,0) \in \Omega$ and $\Omega^{* *}$ be an arbitrary subdomain of $\Omega^{*}$ with $\operatorname{dist}\left(\Omega^{* *}, \partial \Omega^{*}\right)>0$. Then the following estimate holds:

$$
\rho \leq C_{1} \quad \text { for } \xi \in \Omega^{*},
$$

where $C_{1}$ is a constant depending only on $\operatorname{dist}\left(\Omega^{* *}, \partial \Omega^{*}\right), \operatorname{diam}(\Omega)$ and $\left|L_{1}\right|$.

Next we estimate $1 / \rho=\left[\operatorname{det}\left(\partial^{2} f / \partial x_{i} \partial x_{j}\right)\right]^{1 / 4}$ from above. Let $\Omega^{\prime}$ be an arbitrary subdomain of $\Omega$ with $\operatorname{dist}\left(\Omega^{\prime}, \partial \Omega\right)=2 r>0$. It is easy to see that

$$
\left(\sum\left(\frac{\partial f}{\partial x_{i}}\right)^{2}(x)\right)^{1 / 2} \leq \frac{\sup _{y, z \in \Omega}|f(y)-f(z)|}{\operatorname{dist}(x, \partial \Omega)} \quad \text { for } x \in \Omega .
$$


On the other hand, by a similar calculation as in the proof of Lemma 3.3, one can obtain $(3.22)$

$\frac{1}{\rho} \leq \exp \left\{\frac{33}{8}\right\}\left[2^{1 / 4} A^{1 / 4}\left(2^{1 / 4} A^{1 / 4}\left|L_{1}\right|+2^{3 / 2}\right)+2 \exp \{-1 / 2\} \frac{A^{1 / 4}}{r^{1 / 2}}\right] \quad$ for $x \in B_{\frac{1}{2} r}\left(x_{0}\right)$,

where $\bar{B}_{r}\left(x_{0}\right)=\left\{\left(x_{1}, x_{2}\right) \mid \sum\left(x_{i}-x_{i}^{\circ}\right)^{2} \leq r^{2}\right\} \subset \Omega$ and $A=\max _{\bar{B}_{r}\left(x_{0}\right)} \sum\left(\partial f / \partial x_{i}\right)^{2}$. Combination of (3.21) and (3.22) gives us

LEMMA 3.4. Let $\Omega$ be a bounded domain and $\Omega^{\prime}$ be an arbitrary subdomain of $\Omega$ with $\operatorname{dist}\left(\Omega^{\prime}, \partial \Omega\right)>0$. Then the following estimate holds:

$$
\frac{1}{\rho} \leq C_{2} \quad \text { for } x \in \Omega^{\prime}
$$

where $C_{2}$ is a constant depending only on $\operatorname{dist}\left(\Omega^{\prime}, \partial \Omega\right), \sup _{y, z \in \Omega}|f(y)-f(z)|$, and $\left|L_{1}\right|$.

Proof of Theorem 3.1. Suppose that $M$ is a locally strongly convex, Euclidean complete affine surface. Obviously, $M$ is affine complete if $M$ is compact. Therefore, it is enough to consider the case when $M$ is a non-compact, Euclidean complete, locally strongly convex surface with constant affine mean curvature $L_{1}$. From Hadamard's Theorem(see [WU]) $M$ is the graph of a strictly convex function $x_{3}=f\left(x_{1}, x_{2}\right)$ defined in a convex domain $V \subset A^{2}$. To prove Theorem 3.1, we need the following result (see [L-S-Z], p. 117):

THEOREM 3.5. Let $M$ be a locally strongly convex, Euclidean complete hypersurface in $A^{n+1}$. If there is a constant $N>0$ such that

$$
\|B\|_{G}^{2} \leq N
$$

then $M$ is also affine complete.

We now use blow-up analysis to show that there is a constant $N>0$ such that $\|B\|_{G}^{2} \leq N$ everywhere. Then, by Theorem 3.5, Theorem 3.1 follows. To this end, suppose that this is false. Then there would exist a sequence of points $\left\{p_{k}\right\} \subset M$ such that

$$
\|B\|_{G}^{2}\left(p_{k}\right) \rightarrow \infty
$$

as $k \rightarrow \infty$. For each $p_{k} \in M$ we may assume that the plane $x_{3}=0$ is parallel to the tangent plane of $M$ at $p_{k}$ and $p_{k}$ has the coordinates $(0,0)$. With respect to this coordinate system we have $f \geq 0$, and for any number $C>0$ the set

$$
M_{C}=\left\{\left(x_{1}, x_{2}\right) \in V \mid x_{3}=f\left(x_{1}, x_{2}\right)<C\right\}
$$

is a bounded convex domain in $A^{2}$. It is well-known that there exists a unique ellipsoid $E$, which attains the minimum volume among all the ellipsoids that contain $M_{C}$ and that are centered at the center of mass of $M_{C}$, and a positive constant $\alpha$ such that

$$
\alpha E \subset M_{C} \subset E,
$$

where $\alpha E$ means the $\alpha$-dilation of $E$ with respect to its center. By an orthogonal linear transformation, we may suppose that the equation of the minimum ellipsoid $E$ is

$$
\frac{\left(x_{1}-x_{1}^{\circ}\right)^{2}}{a_{1}^{2}}+\frac{\left(x_{2}-x_{2}^{\circ}\right)^{2}}{a_{2}^{2}}=1 .
$$


By the unimodular affine transformation

$$
\widehat{x}_{1}=\sqrt{C \frac{a_{2}}{a_{1}}} x_{1}, \quad \widehat{x}_{2}=\sqrt{C \frac{a_{1}}{a_{2}}} x_{2}, \quad \widehat{x}_{3}=\frac{1}{C} x_{3},
$$

$M$ is given as a graph of a strictly convex function $\widehat{f}\left(\widehat{x}_{1}, \widehat{x}_{2}\right)$ defined in a convex domain $\widehat{\Omega} \subset A^{2}$. Denote by $L_{C}$ the linear transformation

$$
\widehat{x}_{1}=\sqrt{C \frac{a_{2}}{a_{1}}} \cdot x_{1}, \quad \widehat{x}_{2}=\sqrt{C \frac{a_{1}}{a_{2}}} \cdot x_{2} .
$$

Then $L_{C}(E)$ is the ball $B_{r}$ with center $\left(\sqrt{C \frac{a_{2}}{a_{1}}} x_{1}^{\circ}, \sqrt{C \frac{a_{1}}{a_{2}}} x_{2}^{\circ}\right)$ and radius $r=\sqrt{C a_{1} a_{2}}$. Setting

(3.23) becomes

$$
\Omega_{C}=L_{C}\left(M_{C}\right)
$$

Obviously, we have

$$
\alpha B_{r} \subset \Omega_{C} \subset B_{r} .
$$

$$
\Omega_{C}=\left\{\left(\widehat{x}_{1}, \widehat{x}_{2}\right) \in \widehat{\Omega} \mid \widehat{f}\left(\widehat{x}_{1}, \widehat{x}_{2}\right)<1\right\} .
$$

It is easy to see that the function

$$
H:(0, \infty) \rightarrow \mathbb{R}, H(C)=\pi \cdot C \cdot a_{1}(C) a_{2}(C)
$$

is continuous. Note that $H((0, \infty))=(0, \infty)$. It follows that there exists a number $C^{(k)}>0$ such that $C^{(k)} a_{1}\left(C^{(k)}\right) a_{2}\left(C^{(k)}\right)=1$. This implies that, by a unimodular affine transformation (3.25) with $C=C^{(k)}, M$ is given by a strictly convex function $f^{(k)}$ defined in a convex domain in $A^{2}$ such that

$$
B\left(x^{(k)}, \alpha\right) \subset \Omega_{k} \subset B\left(x^{(k)}, 1\right), \quad \text { where } \quad \Omega_{k}=\left\{\left(x_{1}, x_{2}\right) \mid f^{(k)}<1\right\} .
$$

Thus, we would obtain a sequence of convex functions $\left\{f^{(k)}\right\}$ and a sequence of points $\left\{x^{(k)}\right\}$ such that $f^{(k)} \geq 0$, and such that $B\left(x^{(k)}, \alpha\right) \subset \Omega_{k} \subset B\left(x^{(k)}, 1\right)$. Therefore, we may suppose, by taking subsequences, that $\left\{\Omega_{k}\right\}$ converges to a convex domain $\Omega$ and $\left\{f^{(k)}\right\}$ converges to a convex function $f_{\infty}$, locally uniformly in $\Omega$. For $x \in \partial \Omega$, we define $f_{\infty}(x)=\underline{\lim }_{y \rightarrow x, y \in \Omega} f_{\infty}(y)$.

In the following we shall give the uniform estimates of $\operatorname{det}\left(\partial^{2} u^{(k)} / \partial \xi_{i} \partial \xi_{j}\right)$ from below and above (where $u^{(k)}$ denotes the Legendre transformation relative to $f^{(k)}$ ), and use the Caffarelli-Gutierrez theory to obtain a Hölder estimate for $\operatorname{det}\left(\partial^{2} u^{(k)} / \partial \xi_{i} \partial \xi_{j}\right)$ ( see $[\mathrm{C}-\mathrm{G}]$ or $[\mathrm{T}-\mathrm{W}]$, Theorem 4.1$)$. Then we use the Caffarelli-Schauder estimate for the Monge-Ampère equation $[\mathrm{CAF}]$ to get a $C^{2, \alpha}$ estimate to show that the limit surface is a smooth surface.

Estimate for $\operatorname{det}\left(\partial^{2} u^{(k)} / \partial \xi_{i} \partial \xi_{j}\right)$ from below. Let us denote

$$
D=\left\{x \in \bar{\Omega} \mid f_{\infty}(x)=0\right\}
$$

where $\bar{\Omega}$ denotes the closure of $\Omega$. It is easy to see that $D$ is a closed subset of $\bar{\Omega}$ and $(0,0) \in D$. To estimate $\operatorname{det}\left(\partial^{2} u^{(k)} / \partial \xi_{i} \partial \xi_{j}\right)$, we shall consider different cases according to the location of $D$ :

CASE 1: $D \subset \Omega$.

CASE 2: $D \cap \partial \Omega \neq \emptyset$. 
Later we shall prove that Case 2 cannot take place. Now we consider Case 1 . In this case, there exists a number $b, 0<b<1$, such that the set

$$
\bar{\Omega}_{2 b} \subset \Omega,
$$

where $\Omega_{2 b}:=\left\{\left(x_{1}, x_{2}\right) \in \Omega \mid f_{\infty}\left(x_{1}, x_{2}\right)<2 b\right\}$. Put $\Omega_{k, b}:=\left\{\left(x_{1}, x_{2}\right) \in \Omega_{k} \mid f^{(k)}\left(x_{1}, x_{2}\right)\right.$ $<b\}$. Since $\left\{f^{(k)}\right\}$ converges to $f_{\infty}$ locally uniformly,

$$
\bar{\Omega}_{k, b} \subset \overline{\Omega_{2 b}} \subset \Omega
$$

for $k$ large enough; it follows that

$$
\operatorname{dist}\left(\Omega_{k, b}, \partial \Omega_{k}\right)>d
$$

for $k$ large enough, where $d>0$ is a constant independent of $k$. Now we use Lemma 3.2 to conclude that

$$
\operatorname{det}\left(\frac{\partial^{2} f^{(k)}}{\partial x_{i} \partial x_{j}}\right) \leq d_{1} \quad \text { for } x \in \Omega_{k, b},
$$

where $d_{1}>0$ is a constant depending only on $\left|L_{1}\right|$ and $d$. Consider the Legendre transformation relative to $f^{(k)}$ :

$$
\xi_{i}^{(k)}=\frac{\partial f^{(k)}}{\partial x_{i}}, \quad u^{(k)}=\sum x_{i} \frac{\partial f^{(k)}}{\partial x_{i}}-f^{(k)} .
$$

Set

$$
\Omega_{k}^{*}=\left\{\left(\xi_{1}^{(k)}(x), \xi_{2}^{(k)}(x)\right) \mid\left(x_{1}, x_{2}\right) \in \Omega_{k}\right\}, \quad \Omega_{k, b}^{*}=\left\{\left(\xi_{1}^{(k)}(x), \xi_{2}^{(k)}(x)\right) \mid\left(x_{1}, x_{2}\right) \in \Omega_{k, b}\right\} .
$$

Then, by (3.27), we have

$$
\operatorname{det}\left(\frac{\partial^{2} u^{(k)}}{\partial \xi_{i} \partial \xi_{j}}\right) \geq \frac{1}{d_{1}} \quad \text { for } \xi \in \Omega_{k, b}^{*} .
$$

Estimate for $\operatorname{det}\left(\partial^{2} u^{(k)} / \partial \xi_{i} \partial \xi_{j}\right)$ from above. To get the estimate, we need some important results of classical convex body theory (see [BA], [BU]). Let $F$ be a convex hypersurface in $A^{n+1}$ and $e$ be a subset of $F$. We denote by $\psi_{F}(e)$ the spherical image of $e$. If the set $e$ is a Borel set, the spherical image of the set $e$ is also a Borel set and therefore is measurable. Denote by $\sigma_{F}(e)$ the area (measure) of the spherical image $\psi_{F}(e)$ of the Borel set $e$ of $F$ and call it the integral Gaussian curvature of $e$. Denote by $A(e)$ the measure (or area) of the Borel set $e$ on $F$. The ratio $\sigma_{F}(e) / A(e)$ is called the specific curvature of $e$. The following theorems hold (see [BU], [BA]):

TheOrem 3.6 (A. V. Pogorelov). A convex surface whose specific curvature is bounded away from zero is strictly convex.

THEOREM 3.7. Let a sequence of closed convex hypersurfaces $F_{k}$ converge to a closed convex hypersurface $F$ and a sequence of closed subset $M_{k}$ of $F_{k}$ converge to a closed subset $M$ of $F$; then

$$
\sigma_{F}(M) \geq \varlimsup_{k \rightarrow \infty} \sigma_{F_{k}}\left(M_{k}\right) .
$$

First of all, we claim that there exists a ball $B(0, r)$ such that

$$
B(0, r) \subset \Omega_{k, b}^{*} \quad \text { for } k=1,2, \ldots
$$


In fact, since $\Omega$ is bounded, there is a ball $B\left(0, r_{1}\right)$ with the center $(0,0)$ and the radius $r_{1}$ such that

$$
\bar{\Omega}_{b}:=\left\{\left(x_{1}, x_{2}\right) \in \Omega \mid f_{\infty}\left(x_{1}, x_{2}\right) \leq b\right\} \subset B\left(0, r_{1}\right) .
$$

Since $\left\{f^{(k)}\right\}$ converges locally uniformly to $f_{\infty}$ in $\Omega$, we see that

$$
\bar{\Omega}_{k, b / 2}:=\left\{\left(x_{1}, x_{2}\right) \in \Omega_{k} \mid f^{(k)}\left(x_{1}, x_{2}\right) \leq b / 2\right\} \subset B\left(0, r_{1}\right)
$$

for $k$ large enough. Consider the convex cone $K$ with vertex $(0,0)$ and the base

$$
\left\{\left(y_{1}, y_{2}, b / 2\right) \mid\left(y_{1}, y_{2}\right) \in \partial \Omega_{k, b / 2}\right\} \text {. }
$$

Then we have (see [BA], p. 115)

$$
\chi_{f^{(k)}}\left(\Omega_{k, b / 2}\right) \supset \chi_{K}\left(\Omega_{k, b / 2}\right) .
$$

On the other hand, since $\Omega_{k, b / 2} \subset B\left(0, r_{1}\right)$, we see that (see [BA], p.126) $\chi_{K}\left(\Omega_{k, b / 2}\right) \supset$ $B\left(0, b / 2 r_{1}\right)$ and the claim follows.

Next, we want to prove that $u_{\infty}$ is strictly convex at $(0,0)$. In fact, since $u^{(k)}$ are convex and bounded, we may assume by taking subsequences, that $\left\{u^{(k)}\right\}$ converges locally uniformly to a convex function $u_{\infty}$ in $\Omega_{b / 2 r_{1}}^{*}=\left\{\left(\xi_{1}, \xi_{2}\right) \mid \sum \xi_{i}^{2}<b^{2} / 4 r_{1}^{2}\right\}$. Let $e$ be a closed subset of $\Omega_{b / 2 r_{1}}^{*}$ with $e^{0} \neq \emptyset$, where $e^{0}$ denotes the interior of $e$. We denote by $F$ and $F^{(k)}$ the graphs of the functions $u_{\infty}: \Omega_{b / 2 r_{1}}^{*} \rightarrow \mathbb{R}$ and $u^{(k)}: \Omega_{b / 2 r_{1}}^{*} \rightarrow \mathbb{R}$, respectively.

Set

$$
F_{e}=\left\{\left(\xi_{1}, \xi_{2}, u_{\infty}\left(\xi_{1}, \xi_{2}\right)\right) \mid\left(\xi_{1}, \xi_{2}\right) \in e\right\}, \quad F_{e}^{(k)}=\left\{\left(\xi_{1}, \xi_{2}, u^{(k)}\left(\xi_{1}, \xi_{2}\right)\right) \mid\left(\xi_{1}, \xi_{2}\right) \in e\right\} .
$$

Then, by Theorem 3.7, we get

$$
\begin{aligned}
\sigma_{F}\left(F_{e}\right) & \geq \varlimsup_{k \rightarrow \infty} \sigma_{F^{(k)}}\left(F_{e}^{(k)}\right)=\varlimsup_{k \rightarrow \infty} \int_{F_{e}^{(k)}} \frac{\operatorname{det}\left(\frac{\partial^{2} u^{(k)}}{\partial \xi_{i} \partial \xi_{j}}\right)}{\left(1+\sum\left(\frac{\partial u^{(k)}}{\partial \xi_{i}}\right)^{2}\right)^{2}} d p \\
& \geq b_{2} \varlimsup_{k \rightarrow \infty} A\left(F_{e}^{(k)}\right)=b_{2} A\left(F_{e}\right),
\end{aligned}
$$

where $b_{2}$ is a constant depending only $d_{1}$ and $\operatorname{diam}(\Omega)$, i.e.,

$$
\frac{\sigma_{F}\left(F_{e}\right)}{A\left(F_{e}\right)} \geq b_{2}>0 \text {. }
$$

By Theorem 3.6, we conclude that $u_{\infty}$ is strictly convex at $(0,0)$.

We now estimate $\operatorname{det}\left(\partial^{2} u^{(k)} / \partial \xi_{i} \partial x \xi_{j}\right)$ from above. Since $u_{\infty}$ is strictly convex at $(0,0)$, there exists a positive constant $0<h_{1}<1$, such that

$$
\Omega_{h_{1}}^{*}=\left\{\left(\xi_{1}, \xi_{2}\right) \in \Omega_{b / 2 r_{1}}^{*} \mid u_{\infty}\left(\xi_{1}, \xi_{2}\right)<h_{1}\right\}
$$

is a bounded convex domain. Then, we choose $0<h_{2}<h_{1}$ such that

$$
\bar{\Omega}_{2 h_{2}}^{*} \subset \Omega_{h_{1}}^{*},
$$

where $\Omega_{2 h_{2}}^{*}=\left\{\left(\xi_{1}, \xi_{2}\right) \in \Omega_{h_{1}}^{*} \mid u_{\infty}\left(\xi_{1}, \xi_{2}\right)<2 h_{2}\right\}$. Put

$$
\Omega_{k, h_{2}}^{*}=\left\{\left(\xi_{1}, \xi_{2}\right) \in \Omega_{k}^{*} \mid u^{(k)}\left(\xi_{1}, \xi_{2}\right)<h_{2}\right\} .
$$

Since $u^{(k)}$ converges locally uniformly to $u_{\infty}$, we have

$$
\bar{\Omega}_{k, h_{2}}^{*} \subset \Omega_{h_{1}}^{*}
$$


and there exists a constant $d_{2}>0$, such that

$$
\operatorname{dist}\left(\Omega_{k, h_{2}}^{*}, \partial \Omega_{h_{1}}^{*}\right)>d_{2}
$$

for $k$ large enough. Clearly, there is a uniform estimate

$$
\sum\left(\frac{\partial u^{(k)}}{\partial \xi_{i}}\right)^{2} \leq \operatorname{diam}(\Omega) \quad \text { for } \xi \in \Omega_{b / 2 r_{1}}^{*}
$$

Now we use Lemma 3.1 to conclude that

$$
\operatorname{det}\left(\frac{\partial^{2} u^{(k)}}{\partial \xi_{i} \partial \xi_{j}}\right)<d_{3} \quad \text { for } \xi \in \Omega_{k, h_{2}}^{*},
$$

for $k$ large enough, where $d_{3}>0$ is a constant depending only on $\operatorname{diam}(\Omega), d_{2}$ and $\left|L_{1}\right|$.

We are now ready to prove the following lemma:

LEMMA 3.7. There exists a neighborhood $U$ of $(0,0)$ such that

$$
D^{2} u^{(k)} \geq C_{3} I, \quad\left|D^{l} u^{(k)}\right| \leq C_{4} \quad \text { for } k, l=1,2, \ldots,
$$

where $C_{3}$ and $C_{4}$ are constants. $C_{3}$ depends only on $d_{2}, \operatorname{dim}(\Omega),\left|L_{1}\right|$ and $d$ and $C_{4}$ depends additionally on $l$.

Proof. We set

$$
v=\frac{1}{\rho}, \quad \rho_{i}=\frac{\partial \rho}{\partial \xi_{i}}, \quad v_{i}=\frac{\partial v}{\partial \xi_{i}} v_{i j}=\frac{\partial^{2} v}{\partial \xi_{i} \partial \xi_{j}}
$$

Then

$$
\Delta\left(\frac{1}{v}\right)=\frac{1}{\rho} \sum u^{i j}\left(-\frac{v_{i j}}{v^{2}}+2 \frac{v_{i} v_{j}}{v^{3}}\right)-\frac{2}{\rho^{2}} \sum u^{i j} \frac{v_{i} v_{j}}{v^{4}}=-\rho \sum u^{i j} v_{i j}
$$

On the other hand we have $\Delta(1 / v)=\Delta \rho=-n L_{1} \rho$, and it follows that

$$
\sum u^{i j} v_{i j}-n L_{1}=0 \text {. }
$$

Therefore, by setting

$$
\Psi=v-L_{1} u+2\left|L_{1}\right|
$$

we obtain

$$
\sum U^{i j} \Psi_{i j}=0
$$

where $\left(U^{i j}\right)$ is the matrix of cofactors of $\left(u_{i j}\right)$. By (4.6), (4.8) and (4.10), we use the Caffarelli-Gutierrez theory to obtain a Hölder estimate for $\operatorname{det}\left(\partial^{2} u^{(k)} / \partial \xi_{i} \partial \xi_{j}\right)$ (see [C-G] or $[\mathrm{T}-\mathrm{W}]$, Theorem 4.1). Then we use the Caffarelli-Schauder estimate for the MongeAmpère equation $([\mathrm{CAF}])$ to get a $C^{2, \alpha}$ estimate. Finally, by bootstrapping, Lemma 3.7 follows.

Consequently, from Lemma 3.7 it follows that $u_{\infty}$ is a smooth strictly convex function in a neighborhood of $(0,0)$, and hence $f_{\infty}$ is a smooth strictly convex function in a neighborhood of $(0,0)$.

Now it is our purpose to show that Case 2 cannot take place. Let

$$
\begin{gathered}
V_{k}=\left\{\left(x_{1}, x_{2}, x_{3}\right) \in A^{3} \mid f^{(k)}\left(x_{1}, x_{2}\right) \leq x_{3} \leq 1,\left(x_{1}, x_{2}\right) \in \bar{\Omega}_{k}\right\}, \\
V_{\infty}=\left\{\left(x_{1}, x_{2}, x_{3}\right) \in A^{3} \mid f_{\infty}\left(x_{1}, x_{2}\right) \leq x_{3} \leq 1,\left(x_{1}, x_{2}\right) \in \bar{\Omega}\right\} .
\end{gathered}
$$


Then the sequence of convex bodies $\left\{V_{k}\right\}$ converges to the convex body $V_{\infty}$. First we claim that $D$ is a line segment or a single point. To prove this claim, we first show that there exists a ball $B\left(0, r_{0}\right)$ with center $(0,0)$ and the radius $r_{0}$ such that

$$
B\left(0, r_{0}\right) \subset \Omega_{k}^{*} \quad \text { for } k=1,2, \ldots
$$

As before, we choose a ball $B(0, l)$ with the center $(0,0)$ and the radius $l$ such that $\Omega \subset B(0, l)$. Since $\Omega_{k}$ converges to $\Omega$, we see that $\Omega_{k} \subset B(0, l)$, for $k$ large enough. Then it is easy to see that $\Omega_{k}^{*} \supset B(0,1 / l)$ for $k=1,2, \ldots$.

Now we prove our claim. By contradiction let us assume that there exists a ball

$$
B\left(x_{0}, \epsilon\right)=\left\{\left(x_{1}, x_{2}\right) \mid \sum\left(x_{i}-x_{i}^{\circ}\right)^{2}<\epsilon^{2}\right\}
$$

such that $B\left(x_{0}, \epsilon\right) \subset D$. Since $\left\{f^{(k)}\right\}$ converges locally uniformly to $f_{\infty}$, there is a positive number $k_{0}$ such that

$$
0 \leq f^{(k)}(x)<\frac{r_{0}^{2} \epsilon}{8} \quad \text { for } x \in B\left(x_{0}, \epsilon / 2\right) \quad\left(k>k_{0}\right) .
$$

Clearly, there exists a uniform estimate

$$
\sum\left(\frac{\partial f^{(k)}}{\partial x_{i}}\right)^{2}<\frac{r_{0}^{2}}{4} \quad \text { for } x \in B\left(x_{0}, \epsilon / 2\right)\left(k>k_{0}\right) .
$$

Put

$$
\Omega_{k, \epsilon / 2}^{*}=\left\{\left(\xi_{1}^{(k)}(x), \xi_{2}^{(k)}\left(x_{1}\right)\right) \mid x \in B\left(x_{0}, \epsilon / 2\right)\right\} .
$$

Then we have

$$
\Omega_{k, \epsilon / 2}^{*} \subset B\left(0, r_{0} / 2\right) \subset \Omega_{k}^{*} \quad \text { for } k>k_{0} .
$$

Note that $B\left(0, r_{0}\right) \subset \Omega_{k}^{*}$ for $k=1,2, \ldots$ Hence we use Lemma 3.3 to conclude that there exists a constant $d_{4}>0$, depending only on $r_{0}$, $\operatorname{diam}(\Omega)$ and $\left|L_{1}\right|$, such that

$$
\operatorname{det}\left(\frac{\partial^{2} u^{(k)}}{\partial \xi_{i} \partial \xi_{j}}\right)<d_{4} \quad \text { for } \xi \in B\left(0, r_{0} / 2\right) \text {. }
$$

This implies that

$$
\operatorname{det}\left(\frac{\partial^{2} f^{(k)}}{\partial x_{i} \partial x_{j}}\right)>\frac{1}{d_{4}} \quad \text { for } x \in B\left(x_{0}, \epsilon / 2\right)\left(k>k_{0}\right) \text {. }
$$

Therefore we can apply the argument of Case 1 to $\left\{f^{(k)}\right\}$ and conclude that the function $f_{\infty}$ is strictly convex at $x_{0}$. This contradiction shows that $D$ must be a line segment or a single point.

Now we prove that Case 2 cannot take place. We shall consider the following two cases:

CASE 2.1: $D \cap \partial \Omega$ contains at most two points.

CAsE 2.2: $D$ is a line segment with $D \subset \partial \Omega$.

CAsE 2.1. Let $p \in D \cap \partial \Omega$ and let $l$ be a supporting line of $\Omega$ at $p$. The line $l$ and the unit normal $\nu$ of the $\left(x_{1}, x_{2}\right)$-plane determine a plane. We denote the plane by $P$. Then the plane $P$ and the $\left(x_{1}, x_{2}\right)$-plane divide the space $\mathbb{R}^{3}$ into four closed subsets such that $V_{\infty}$ lies completely in one of them. Let $\alpha$ be a supporting plane of $V_{\infty}$ containing the line $l$ such that it intersects $P$ and forms an angle $\angle(\alpha, P)=\theta$ with $P$, where 
$\theta>0$ is sufficiently small. Since $p \in \partial V_{\infty}$ and $\alpha$ is a supporting plane of $V_{\infty}$, there is a neighborhood $U \subset \partial V_{\infty}$ which projects orthogonally and one-to-one onto a convex domain $\Omega^{(1)} \subset \alpha$. This implies that, near the point $p, \partial V_{\infty}$ can be represented as a graph of a convex function $g$ defined in $\Omega^{(1)}$. Obviously, $g$ is strictly convex at $p$, but is not smooth at $p$. We choose a number $b, 0<b<1$, such that

$$
\Omega^{(2)}=\left\{\left(y_{1}, y_{2}\right) \in \Omega^{(1)} \mid g\left(y_{1}, y_{2}\right)<b\right\}
$$

is a bounded convex domain in $A^{2}$. Then we choose a new coordinate system $\left\{y_{1}, y_{2}, y_{3}\right\}$ such that

1) $p$ has coordinate $(0,0,1)$;

2) the equation of $\alpha$ is $y_{3}=1$.

Since the sequence of convex bodies $\left\{V_{k}\right\}$ converges to the convex body $V_{\infty}$, we see that the boundary $\partial V_{k}$ of $V_{k}$ can also be represented as a graph of a convex function $g^{(k)}$ for sufficiently large $k$. Obviously, $g^{(k)} \rightarrow g+1$ in a bounded convex domain $\Omega^{(3)}$. Note that the graph of $g^{(k)}$ is a locally strongly convex surface with constant affine mean curvature $L_{1}$. Therefore we can apply the argument of Case 1 to $\left\{g^{(k)}\right\}$ and conclude that the function $g$ is a smooth function near the point $p$. The contradiction shows that Case 2.1 cannot take place.

CAse 2.2. In this case, we have $p=(0,0) \in \partial \Omega$. Let $l$ be the line containing $D$. We choose a new coordinate system $\left\{y_{1}, y_{2}, y_{3}\right\}$ as in Case 2.1. Then the boundary $\partial V_{\infty}$ of $V_{\infty}$ can be represented as the graph of a convex function $g$ defined in a convex domain $\Omega^{(3)}$. With respect to this coordinate system we have $g \geq 1$. The boundary $\partial V_{k}$ of $V_{k}$ can also be represented as the graph of a convex function $g^{(k)}$ for sufficiently large $k$. Obviously, $g^{(k)} \rightarrow g$ in $\Omega^{(3)}$. Note that the graph of $g^{(k)}$ is a locally strongly convex surface with constant affine mean curvature $L_{1}$. Again, we shall consider different cases according to the location of

$$
D^{\#}=\left\{\left(y_{1}, y_{2}\right) \in \bar{\Omega}^{(3)} \mid g\left(y_{1}, y_{2}\right)=1\right\} .
$$

CASE 2.2.1: $D^{\#} \subset \Omega^{(3)}$.

CASE 2.2.2. $D^{\#} \cap \partial \Omega^{(3)} \neq \emptyset$.

CASE 2.2.1. Note that $D^{\#}$ is a line segment. Since $D^{\#} \subset \Omega^{(3)}$, we can apply the argument of Case 1 to Case 2.2.1 and conclude that Case 2.2.1 cannot take place.

CASE 2.2.2. In this case, $D^{\#} \cap \partial \Omega^{(3)}$ contains at most two points. Therefore we can apply the argument of Case 2.1 to Case 2.2.2 and conclude that Case 2.2.2 cannot take place.

We are now in a position to prove that $\|B\|_{G}^{2}$ is bounded. By Lemma 3.7, we have

$$
D^{2} u^{(k)} \geq C_{3} I, \quad\left|D^{l} u^{(k)}\right| \leq C_{4}, \quad l=0,1,2, \ldots,
$$

in a neighborhood $U$ of $(0,0)$, where $C_{3}$ and $C_{4}$ are constants. $C_{3}$ depends only on $d, d_{2}, \operatorname{diam}(\Omega)$ and $\left|L_{1}\right|$, and $C_{4}$ depends additionally on $l$. Note that $\|B\|_{G}^{2}$ is equiaffinely invariant. By (3.6) we have

$$
\|B\|_{G}^{2}\left(p_{k}\right)=\left.\sum G_{(k)}^{i s} G_{(k)}^{j t} B_{i j}^{(k)} B_{s t}^{(k)}\right|_{(0,0)},
$$


where

$$
\begin{gathered}
B_{i j}^{(k)}=-\frac{1}{\rho^{(k)}} \frac{\partial^{2} \rho^{(k)}}{\partial x_{i} \partial x_{j}}+\frac{2}{\left(\rho^{(k)}\right)^{2}} \frac{\partial \rho^{(k)}}{\partial x_{i}} \frac{\partial \rho^{(k)}}{\partial x_{j}}+\sum \frac{f^{(k) s t}}{\rho^{(k)}} \frac{\partial \rho^{(k)}}{\partial x_{s}} \frac{\partial f_{i j}^{(k)}}{\partial x_{t}} . \\
\rho^{(k)}=\left[\operatorname{det}\left(\frac{\partial^{2} f^{(k)}}{\partial x_{i} \partial x_{j}}\right)\right]^{-\frac{1}{4}}, \quad G_{i j}^{(k)}=\rho^{(k)} \frac{\partial^{2} f^{(k)}}{\partial x_{i} \partial x_{j}} .
\end{gathered}
$$

Note that

$$
\begin{aligned}
\frac{\partial^{2} \rho^{(k)}}{\partial x_{i} \partial x_{j}} & =\sum \frac{\partial^{2} \rho^{(k)}}{\partial \xi_{l} \partial \xi_{s}} u^{(k) l i} u^{(k) s j}+\sum \frac{\partial \rho^{(k)}}{\partial \xi_{l}} \frac{\partial u^{(k) l i}}{\partial \xi_{s}} u^{(k) s j} \\
\frac{\partial \rho^{(k)}}{\partial x_{i}} & =\sum \frac{\partial \rho^{(k)}}{\partial \xi_{l}} u^{(k) l i}, \quad \frac{\partial f_{i j}^{(k)}}{\partial x_{l}}=\sum \frac{\partial u^{(k) i j}}{\partial \xi_{s}} u^{(k) s l} .
\end{aligned}
$$

Consequently, from Lemma 3.7, it follows that there exists a number $N>0$ such that

$$
\|B\|_{G}^{2}\left(p_{k}\right) \leq N, \quad k=1,2, \ldots
$$

On the other hand, by (3.23) we have

$$
\|B\|_{G}^{2}\left(p_{k}\right) \rightarrow \infty \quad \text { as } k \rightarrow \infty .
$$

The contradiction shows that there must exist a number $N>0$ such that $\|B\|_{G}^{2} \leq N$ on $M$. Then, by Theorem 3.5, Theorem 3.1 follows.

Proof of Theorem 3.2. Recall that (see [L-S-Z], p. 121) there is no locally strongly convex, compact hypersurface without boundary and with non-positive affine mean curvature. This implies that $M$ is non-compact if $M$ is a locally strongly convex surface with constant affine curvature $L_{1} \leq 0$.

(a) Denote by $R$ the scalar curvature; we have $R=2\left(J+L_{1}\right) \geq 2 L_{1}>0$ (see [L-S-Z], p. 76). Moreover, by Theorem 3.1, $M$ is affine-complete. This implies that $(M, G)$ is a complete Riemannian manifold with Ricci curvature bounded from below by a positive constant $2 L_{1}>0$. By Myer's and Bonnet's Theorem (see [CH-E], p. 27) $M$ is compact. It follows that $M$ is an ellipsoid see ([L-S-Z], p. 121).

(b) Since $M$ is an Euclidean complete and affine complete affine maximal surface, by Calabi's Theorem (see, [L-S-Z], p. 219) $M$ must be an elliptic paraboloid.

(c) Since the tensor norm is equiaffinely invariant, we replace $\|B\|_{G}^{2}$ by it, then a similar argument shows that the tensor norms of the Fubini-Pick tensor and the affine Weingarten tensor and tensor norms of the $k$-th covariant differentiation of them are all bounded. This completes the proof of Theorem 3.2.

\section{References}

[BA] I. J. Bakelman, Convex Analysis and Nonlinear Geometric Elliptic Equations, SpringerVerlag, Berlin, 1994.

[BU] H. Busemann, Convex Surfaces, Interscience Publ., New York-London, 1958.

[CA-1] E. Calabi, Hypersurfaces with maximal affinely invariant area, Amer. J. Math. 104 (1984), 91-126. 
[CA-2] E. Calabi, Convex affine maximal surfaces, Results Math. 13 (1988), 209-223.

[CA-3] E. Calabi, Improper affine hyperspheres of convex type and a generalization of a theorem by K. Joergens, Michigan Math. J. 5 (1958), 105-126.

[CA-4] E. Calabi, Affine differential geometry and holomorphic curves, in: Lecture Notes Math. 1422, Springer, 1990, 15-21.

[CAF] L. A. Caffarelli, Interior $W^{2, p}$ estimates for solutions of Monge-Ampère equations, Ann. Math. 131 (1990), 135-150.

[C-G] L. A. Caffarelli and C. E. Gutierrez, Properties of the solutions of the linearized MongeAmpère equations, Amer. J. Math. 119 (1997), 423-465.

$[\mathrm{CH}] \quad$ S. S. Chern, Affine minimal hypersurfaces, Proc. Japan-U.S. Semin., Tokyo, 1977 (1978), 17-30.

[CH-E] J. Cheeger and D. G. Ebin, Comparison Theorems in Riemannian Geometry, NorthHolland Publishing Company, 1975.

[C-Y] S. Y. Cheng and S. T. Yau, Complete affine hypersurface, Part 1, The completeness of affine metrics, Commun. Pure and Appl. Math. 39 (1986), 839-866.

[L1] A.-M. Li, Affine completeness and Euclidean completeness, in: Lecture Notes in Math. 1481, Springer, 1991, 116-126.

[L2] A.-M. Li, Some theorems in affine differential geometry, Acta Mathematica Sinica, New Series 5, 345-354.

[L-J-1] A.-M. Li and F. Jia, The Calabi conjecture on affine maximal surfaces, Results Math. 40 (2001), 265-272.

[L-J-2] A.-M. Li and F. Jia, Euclidean complete affine hypersurfaces with constant affine mean curvature, Annals of Global Analysis and Geometry 23 (2003), 283-304

[L-S-Z] A.-M. Li, U. Simon and G. S. Zhao, Global Affine Differential Geometry of Hypersurfaces, Walter de Gruyter, Berlin, New York, 1993.

[M] A. Martinez and F. Milan, On the affine Bernstein problem, Geom. Dedicata 37 (1991), 295-302.

[NO] K. Nomizu, On completeness in affine differential geometry, Geom. Dedicata 20 (1986), 43-49.

[N-P-S] K. Nomizu, U. Pinkall and U. Simon (eds.), Affine Differential Geometry, Proc. Math. Forschungsinstitut Oberwolfach, 1991.

[P1] A. V. Pogorelov, On the improper convex affine hyperspheres, Geom. Dedicata 1 (1972), 33-46.

[P2] A. V. Pogorelov, Complete affine minimal hypersurfaces, Soviet Math. Dokl. 38 (1989), $217-219$.

[S1] U. Simon, Hypersurfaces in equiaffine differential geometry, Geom. Dedicata 17 (1984), $157-168$.

[S2] U. Simon (ed.), Affine Differential Geometry, TU Berlin, 1988.

[SCH] R. Schneider, Zur affine Differentialgeometrie im Großen, I. Math. Z. 101 (1967), 375406.

[T] C.-L. Terng, Affine minimal surfaces, Ann. Math. Stud. 103 (1983), 207-216.

[T-W-1] N. Trudinger and X. Wang, The Bernstein problem for affine maximal hypersurfaces, Invent. Math. 140 (2000) 399-422.

[T-W-2] N. Trudinger and X. Wang, Affine complete locally convex hypersurfaces, Invent. Math. 140 (2002), 399-422.

[WU] H. H. Wu, The Spherical images of convex hypersurfaces, J. Diff. Geo. 9 (1974), 279-290. 